\title{
Electrical Properties of Silicon Nanowires Schottky Barriers Prepared by MACE at Different Etching Time
}

\section{Ahlem Rouis ( $\nabla$ rouisahlem@gmail.com )}

Universite de Monastir Faculte des Sciences de Monastir https://orcid.org/0000-0002-9480-061X

\section{Neila Hizem}

Universite de Monastir Faculte des Sciences de Monastir

\section{Mohamed Hassen}

Institut Supérieur des Sciences Appliquées et de Technologie de Sousse: Institut Superieur des Sciences Appliquees et de Technologie de Sousse

Adel kalboussi

Universite de Monastir Faculte des Sciences de Monastir

\section{Original Research}

Keywords: Electrical Properties of Silicon, Etching Time, symmetrical current-voltage, capacitance-voltage

Posted Date: February 11th, 2021

DOI: https://doi.org/10.21203/rs.3.rs-185736/v1

License: (c) (i) This work is licensed under a Creative Commons Attribution 4.0 International License. Read Full License 


\title{
Electrical properties of silicon nanowires Schottky barriers prepared by MACE at different etching time
}

\author{
Ahlem Rouis ${ }^{1, *}$, Neila Hizem¹, Mohamed Hassen², and Adel kalboussi ${ }^{1}$. \\ ${ }^{1}$ Laboratory of Microelectronics and Instrumentation (LR13ES12), Faculty of Science of Monastir, Avenue of \\ Environment, University of Monastir, 5019 Monastir, Tunisia. \\ ${ }^{2}$ Higher Institute of Applied Sciences and Technology of Sousse, Taffala City (Ibn Khaldoun), 4003 Sousse, \\ Tunisia. \\ *Address correspondence to E-mail: rouisahlem@gmail.com
}

\section{ABSTRACT}

This article focused on the electrical characterization of silicon nanowires Schottky barriers following structural analysis of nanowires grown on p-type silicon by Metal (Ag) Assisted Chemical Etching (MACE) method distinguished by their different etching time (5min, 10min, 25min). The SiNWs are well aligned and distributed almost uniformly over the surface of a silicon wafer. In order to enable electrical measurement on the silicon nanowires device, Schottky barriers were performed by depositing Al on the vertically aligned SiNWs arrays. The electrical properties of the resulting Al/SiNWs diodes were characterized by current voltage (I-V) and capacity voltage $(\mathrm{C}-\mathrm{V})$ measurements. Unlike the conventional Schottky diode, symmetrical current-voltage (IV) characteristics have been observed with a rectification ratio $<4$. The metal-semiconductor-metal (M-S-M) model was used to analyze the (I-V) characteristics by including two Schottky barriers at the interface between metal and SiNWs. The electron transport behavior is explained by the thermionic field emission method (TFE) which added the effect of the tunneling current compared to the conventional thermionic emission theory. The capacitance-voltage $\mathrm{C}-\mathrm{V}$ characteristics of SiNWs depend on the bias voltage showing that the samples have an obvious space charge region. Symmetric behavior also appears in the $\mathrm{C}-\mathrm{V}$ curves that confirm the MSM model.

\section{Introduction}

With the advancement of technology within the field of semiconductor, nano miniaturization has emerged bent to be the need for the expansion of electronic industry. Among several nanoelectronic devices, nanowires emerged as a promising candidate for electronic and optical devices like light-emitting diodes (LEDs) [1], photovoltaic devices [2], photodetectors [3], field-effect transistors (FETs) [4], and sensors [5,6]. In recent years, several researchers and industrials have gradually turned their attention to silicon nanowires (SiNWs). Indeed, SiNWs device has been one among promising candidates for integrated circuit technology thanks to their compatibility with well-developed Si-based technology. The large surface-to-volume ratio of nanowires, surface states, such as dangling bonds, and defects, invariably play a dominant role in the electrical and optical characteristics. However, these properties depend on the fabrication technique since it is necessary to control the growth, the structure, and the dimensions of the nanowires.

The metal-assisted chemical etching MACE of Si provides a simple and low-cost approach that enables the fabrication of large-area SiNWs with uniform and controllable height [7, 8]. However, difficulties appeared 
during the production of electrical devices based on silicon nanowires such as the choice of metal for the Ohmic and Schottky contacts, which limited their application. Currently, Ti, Ni, Al, Au are widely used as test electrodes to study the electrical transport properties in SiNWs [9, 10]. For the present experiment, aluminum was chosen to establish Ohmic and Schottky contacts.

The aim of our study is to realize Al / SiNWs Schottky barriers in a first step and to determine the electrical parameters of these structures by I (V) and C (V) measurements in a second step, the relationship between the electrical characteristics SiNWs and the nanowires length was also highlighted.

\section{Experimental details}

The metal (Ag) assisted chemical etching (MACE) method has been wont to fabricate SiNWs. The substrates used here are Czochralski $(\mathrm{Cz})$ solar grade, (100) oriented, p-type boron doped Si wafers, having a

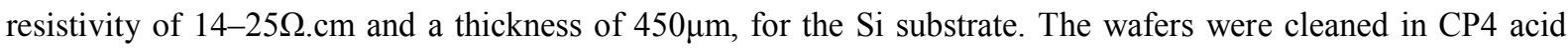
solution (HNO3:64\%, HF: 16\%, $\mathrm{CH} 3 \mathrm{COOH}: 20 \%$ ) for 30 seconds, then rinsed with deionized water and dried. This step had removed approximately $10-15 \mu \mathrm{m}$ of Si from each side. The samples were immersed in a solution of 4.8 MHF (40\%) and 0.02 MAgNO3 for $60 \mathrm{~s}$ to make Ag nanoparticles dendrites films. Then, the Ag-

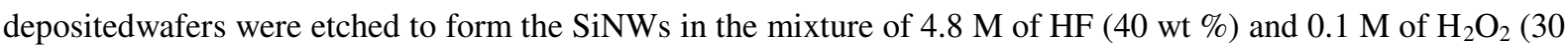
wt \%) solution for $5 \mathrm{~min}$. Subsequently, the as-prepared dark-brown samples were immersed in concentrated aqua fortis $\left(\mathrm{HNO}_{3}\right)$ during $5 \mathrm{~min}$ to get rid of silver particles, rinsed copiously with deionized water and dried. Roughened samples are different by the etching time of the nanowires $(5,10$, and $25 \mathrm{~min})$. The expansion rate of SiNWs is found to be dependent not linearly on the etching time, the estimated lengths are $2.5,5,12.5 \mu \mathrm{m}$ correspondingly $[11,12]$. These samples are compared to a reference (Si-Ref)where the p-Si substrate was not treated neither annealed. First, the as-fabricated SiNWs arrays were tested by scanning electron microscope (SEM).As observed in Fig .1, the SEM images showed well-aligned nanowires in the vertical direction and distributed almost uniformly over the surface of a silicon wafer.

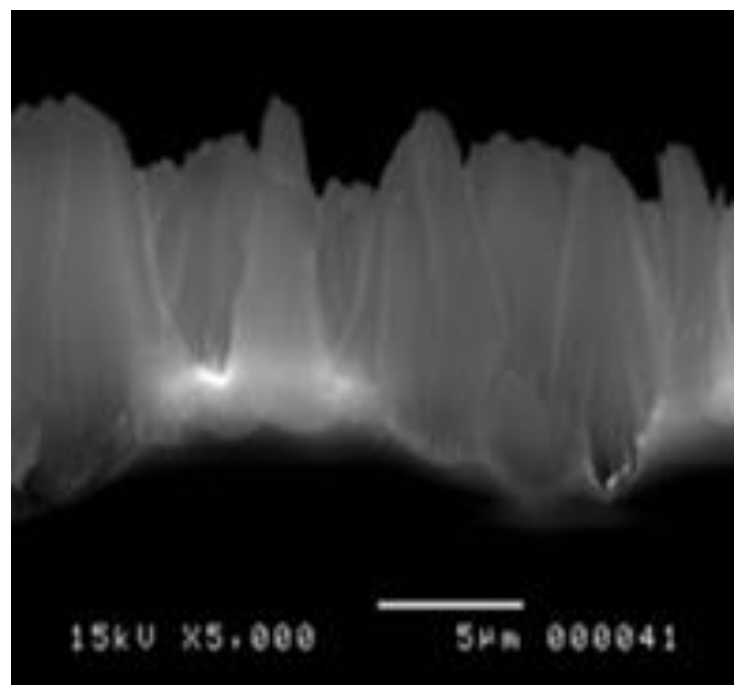

Fig. 1 Cross section SEM images of the silicon nanowires

Second, to understand the electrical study and to determine the mechanism of electrical transport in nanowires, Schottky junctions are formed by depositing a layer of aluminum $300 \mathrm{~nm}$ thick and annealing at 200 
${ }^{\circ} \mathrm{C}$ for the rear Ohmic contact. A circular perforated mask of about $1 \mathrm{~mm}$ in diameter was used to evaporate 100 $\mathrm{nm}$ of aluminum then annealed at $550^{\circ} \mathrm{C}$ to form the Schottky contacts. It was estimated that nanowires made up about $50 \%$ of the Schottky area (Fig.2).

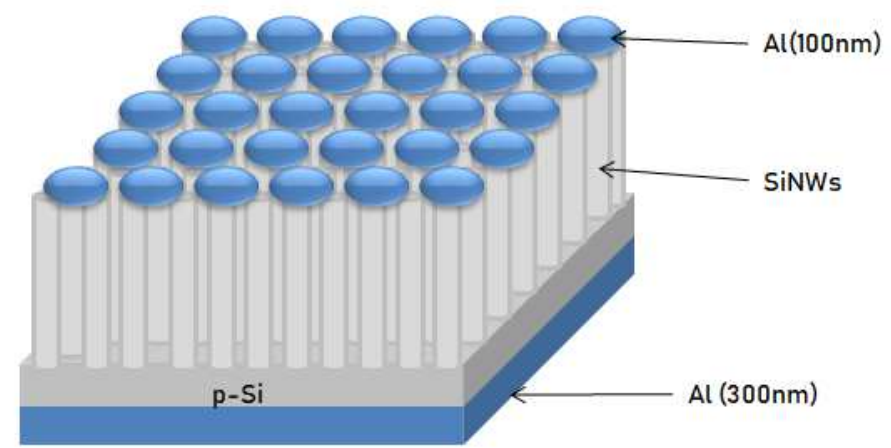

Fig.2 Schematic illustration of the device used in the $\mathrm{I}-\mathrm{V}$ and $\mathrm{C}-\mathrm{V}$ measurements

\section{Results and discussion}

The exponential behavior of $(\mathrm{I}-\mathrm{V})$ characteristics depends on the property of active material used in the device under investigation. Rectifying behavior is expected to see for the current-voltage characteristic of a Schottky Al/SiNWs contact (Al has a high work function of $4.27 \mathrm{eV}$ and Si has an electronic affinity of $3.9 \mathrm{eV}$ [13]).

Current-voltage (I-V) characteristics of the Al/SiNWs etched at different times and Al/Si structures were investigated between the potential ranges from -1 to $+1 \mathrm{~V}$ in dark and at the room temperature and plotted in Fig.3.

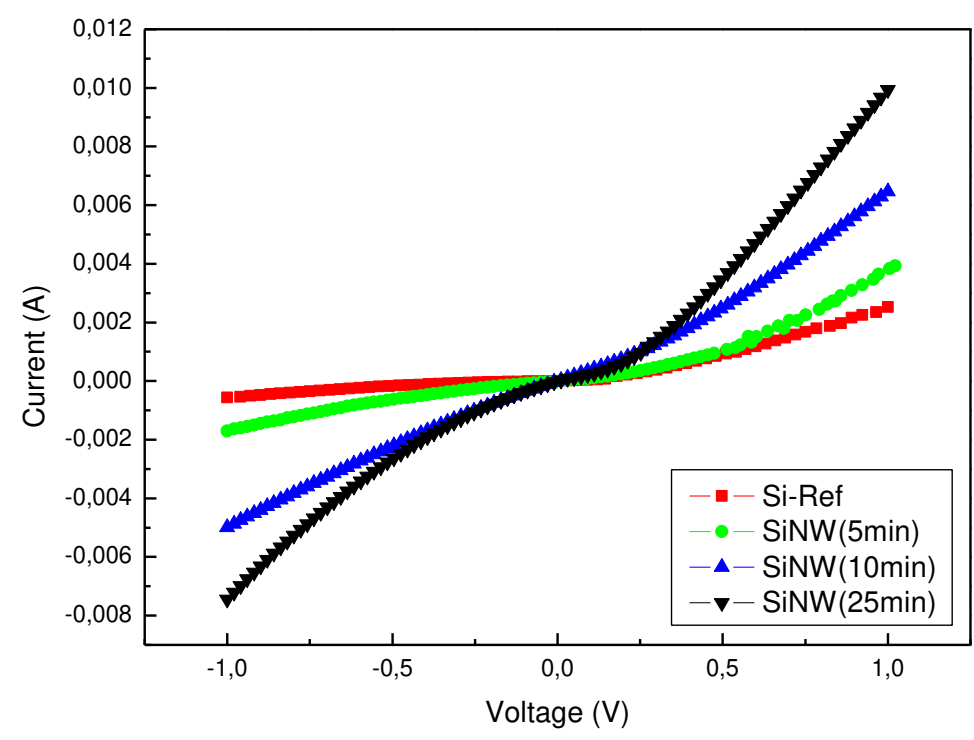

Fig. 3 Experimental I-V characteristics of the Si substrate as reference sample and SiNWs structures obtained in dark and at room temperature. 
As could be seen from Fig. 3, the forward current of a SiNWs is higher than that of a Si-Ref sample and it rises with increasing the nanowire length. The reverse current increases and don't saturate with the applied reverse bias and it increases rapidly in SiNWs samples when increasing the nanowire length. The rectifying ratio is poor and decreases when the length of the nanowire increases $(\mathrm{RR}<4.5$ at $\pm 1 \mathrm{~V})$.

First, the electrical parameters were calculated using the thermionic emission (TE) theory[14, 15].

$$
\mathrm{I}=\mathrm{I}_{\mathrm{S}}\left[\exp \frac{q}{\mathrm{n} K_{B} T}\left(\mathrm{~V}-\mathrm{I} \mathrm{R}_{S}\right)\right]
$$

Where $\mathrm{V}$ is the applied voltage, IRs is voltage drop caused by serial resistance and $\mathrm{K}_{\mathrm{B}}$ is the Boltzmann constant $\mathrm{q}$ is the charge of the electron, $\mathrm{T}$ is the temperature in Kelvin.

Saturation current $\mathrm{I}_{\mathrm{s}}$ is given by the relation:

$$
\mathrm{I}_{\mathrm{S}}=\mathrm{AA}^{*} \mathrm{~T}^{2} \exp \left(\frac{-\mathrm{q} \Phi_{\mathrm{b}}}{\mathrm{K}_{\mathrm{B}} \mathrm{T}}\right)
$$

Where $\mathrm{A} *$ the Richardson constant which is equal to $32 \mathrm{~A} \mathrm{~cm}^{-2} \mathrm{~K}^{-2}$ for $\mathrm{p}-\mathrm{Si}, \mathrm{A}$ is diode area and $\Phi_{\mathrm{B}}$ is the barrier height.

nis the ideality factor used to evaluate the quality of the semiconductor metal interface and to confirm whether the diode transport mechanism is pure TE or not.

$$
\mathrm{n}=\frac{q}{K_{B} T}\left(\frac{d V}{d \ln I}\right) .
$$

The ideality factor extracted from the slope of $\log (\mathrm{I})-\mathrm{V}$ plot(Fig.4) and putted in Table 1, is in range 2-3.25, this high value has also been observed by other works on nanowires [16]. The underlying cause for $n>1$ can be attributed to either the existence of interfacial states or the presence of other transport mechanism like trap assisted tunneling effect in addition to TE [17]. In general the most frequently observed (I-V) characteristics from a semiconducting nanowires is neither linear nor rectifying instead being an almost symmetric I-V characteristic.It can be seen that our structures behave like metal-semiconductor-metal (M-S-M) where the metal-semiconductor contacts in both sides are treated as a Schottky barrier. The M-S-M structure may therefore be modeled as being composed of two Schottky barriers connected back to back in series with a semiconductor resistance [18]. As reported by Z.Y. Zhang et al [19], when the structure M-S-M is polarized there is always Schottky barrier 1 is forward biased and Schottky barrier 2 is reverse biased, the current is thermionic for Schottky barrier 1 and thermionic field emission for Schottky barrier 2. 


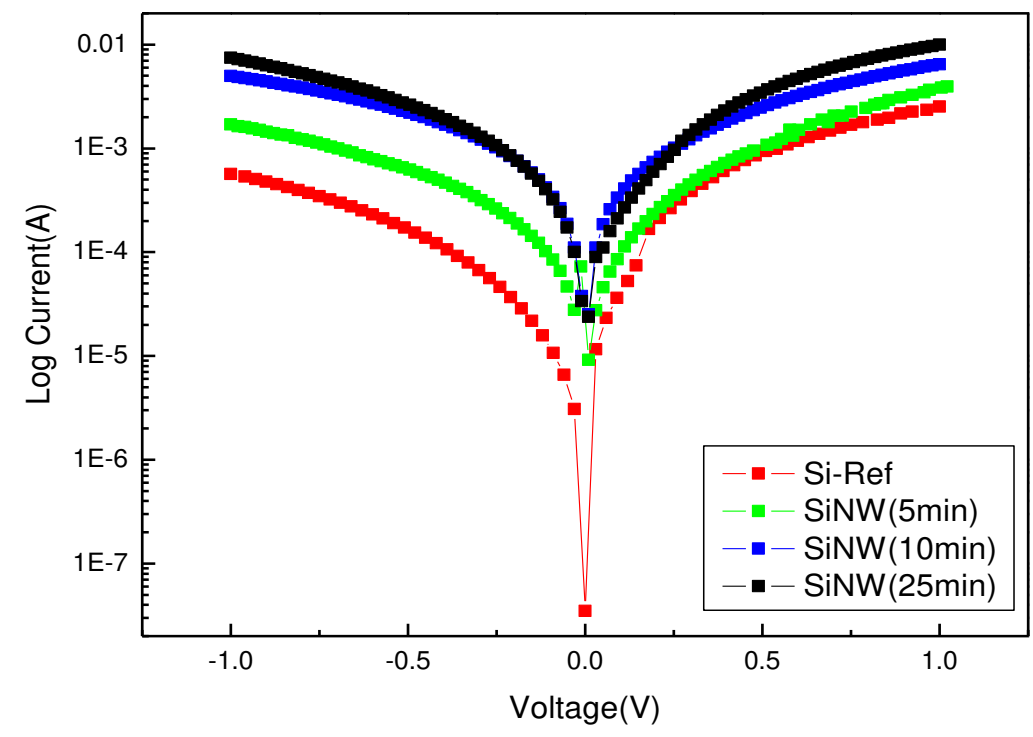

Fig. 4 Experimental semi-logarithmic logI-V characteristics of the c-Si as reference sample and SiNWs structures at room temperature.

Figure 4, shows the semi-logarithmic forward and reverse bias I-V plots of the SiNWs and the Si-Ref diodes. The reverse current increases rapidly in nano Schottky barriers, the tunneling current is therefore not negligible and then becomes the dominating mechanism under reverse bias especially in low dimensional systems. Consequently, for reverse-bias voltage the tunneling current is mainly the thermionic field emission (TFE) current as given in the following equations.

$$
I_{S r}=\frac{A^{*} T\left(\pi \mathrm{q} \mathrm{E}_{00}\right)^{1 / 2}}{k} \exp \left(-\frac{\Phi_{b}}{q E_{0}}\right) \times\left\{q(V-\zeta)+\frac{\Phi_{\mathrm{b}}}{\cosh ^{2}\left(\frac{\mathrm{qE}_{00}}{\mathrm{kT}}\right)}\right\}^{1 / 2}
$$

Where $\zeta$ is the distance between the Fermi energy to the bottom of the conduction band.

$$
E_{0}=E_{00} \operatorname{coth}\left(\frac{q E_{00}}{k T}\right)
$$

With

$$
E_{00}=\frac{h}{2}\left[\frac{N_{A}}{m_{\square}^{*} \varepsilon_{S} \varepsilon_{0}}\right]
$$

$E_{00}$ is a very important parameter in tunneling theory in which $N_{A}$ is the acceptor density at the metal/semiconductor interface, $m_{\square}^{*}$ and $\varepsilon_{s}$ being the effective mass of electron and relative permittivity of the semiconducting nanowire respectively, and $\varepsilon_{0}$ is the permittivity of free space.

The barrier height $\Phi_{\mathrm{b}}$ calculated using the TFE theory (Eqs. 4-6) is in range 0.54 and $0.7 \mathrm{eV}$. These values are reported in Table 1 , they are close to other reports $(0.6-0.8 \mathrm{eV})$ for the configuration of $\mathrm{Al} / \mathrm{TiO}_{2} / \mathrm{p}-\mathrm{Si}$ [16].While, compared to the difference between the working function of metal (Al) and p-type semiconductor nanowire $(\sim 0.73 \mathrm{eV})$, it turns out to be slightly lower, this decrease in barrier height is probably due to the presence of interfacial states and a force-image.Indeed, due to the boundary conditions at the metal surface, the 
charges in the space charge layer will induce image charges in the metal. The interaction with these image charges effectively lowers the barrier height by an amount $\Delta \varphi[20]$.

According to the TFE theory, the specific contact resistance $\mathrm{R}_{\mathrm{c}}$ of SiNWs is defined at high voltage by the following expression.

$$
\mathrm{R}_{\mathrm{c}}=\left(\frac{\partial J}{\partial V}\right)_{V=0}^{-1}=\frac{E_{00}}{q \cdot J_{s r}}
$$

The specific contact resistance $\mathrm{R}_{\mathrm{c}}$ defines the electrical properties of the metal-SC interface. The calculated values of $R_{c}$ is between 100 and $600 \Omega$ for Si-ref and SiNWs with slightly lower values for the reference sample compared to those of the device between $\mathrm{Al} / \mathrm{SiNW}$ contacts. The SiNWs have a thicker oxide layer due to the rough surface and thus their contact resistances are greater. The oxide layer on the surface of the SiNWs reduces the electron transmission probability, which presents as contact resistance [21].On the basis of the MSM structure, all electric parameters are calculated by the (I-V) method for the forward and reverse bias and placed in Table 1. It can be seen from Table 1 that the values are similar for both forward and reverse bias which confirms the symmetrical behavior of the double hetero-junctions.

This electrical characterization shows the performance of SiNWs prepared with the MACE method possess coherent electrical transport properties with their corresponding starting wafer Si-Ref. Some differences possibly result from the surface effect of the nanostructure or the etching time, which affects the morphology of SiNWs arrays.

Table1 Electrical parameters obtained from I-V measurements of Al/SiNWs/p-Si/Aldevices at room temperature in dark.

\begin{tabular}{cccccccc}
\hline Samples & Rectifying & \multicolumn{3}{c}{ Forward bias } & \multicolumn{3}{c}{ Reserve bias } \\
\cline { 2 - 7 } & Ratio & $\mathrm{n}$ & $\mathrm{R}_{\mathrm{c}}(\Omega)$ & $\Phi_{\mathrm{b}}(\mathrm{eV})$ & $\mathrm{n}$ & $\mathrm{R}_{\mathrm{c}}(\Omega)$ & $\Phi_{\mathrm{b}}(\mathrm{eV})$ \\
Si-Ref & 4.47 & 2 & 100 & 0.54 & 1.21 & 150 & 0.51 \\
SiNW (5min) & 2.25 & 2.6 & 250 & 0.58 & 2.72 & 325 & 0.53 \\
SiNW (10min) & 1.29 & 3.25 & 430 & 0.62 & 3.65 & 450 & 0.57 \\
SiNW (25 min) & 1.33 & 4.1 & 600 & 0.7 & 3.9 & 590 & 0.67 \\
\hline
\end{tabular}

The capacitance-voltage $(\mathrm{C}-\mathrm{V})$ technique is a convenient method to study some of the semiconductor parameters including barrier height and carrier concentration. Figure 5shows the room temperature $(\mathrm{C}-\mathrm{V})$ measurement of the Al/SiNWs and Si-Ref diodes conducted at 1 MHz.As can be seen from Fig.5, the capacitance plot is dependent on the bias voltage giving an anomalous peak at zero bias, such that peak magnitude decreases with increasing the nanowire length (SiNWs) in comparison to the Si-Ref device due to the surface quality, the existence of interface states, and the re-structuring and re-ordering of surface charge under the etching effect. The $(\mathrm{C}-\mathrm{V})$ curves depict also usual behavior of a metal-semiconductor-metal (MSM) device, namely, the capacitance of the devices decreases as the amplitude of both positive and negative voltages increases, which is mainly due to the increase of the space charge region width of the reverse-biased Schottky diode in the loop. These symmetric $(\mathrm{C}-\mathrm{V})$ characteristics confirm what has been seen in (I-V) characteristics. 


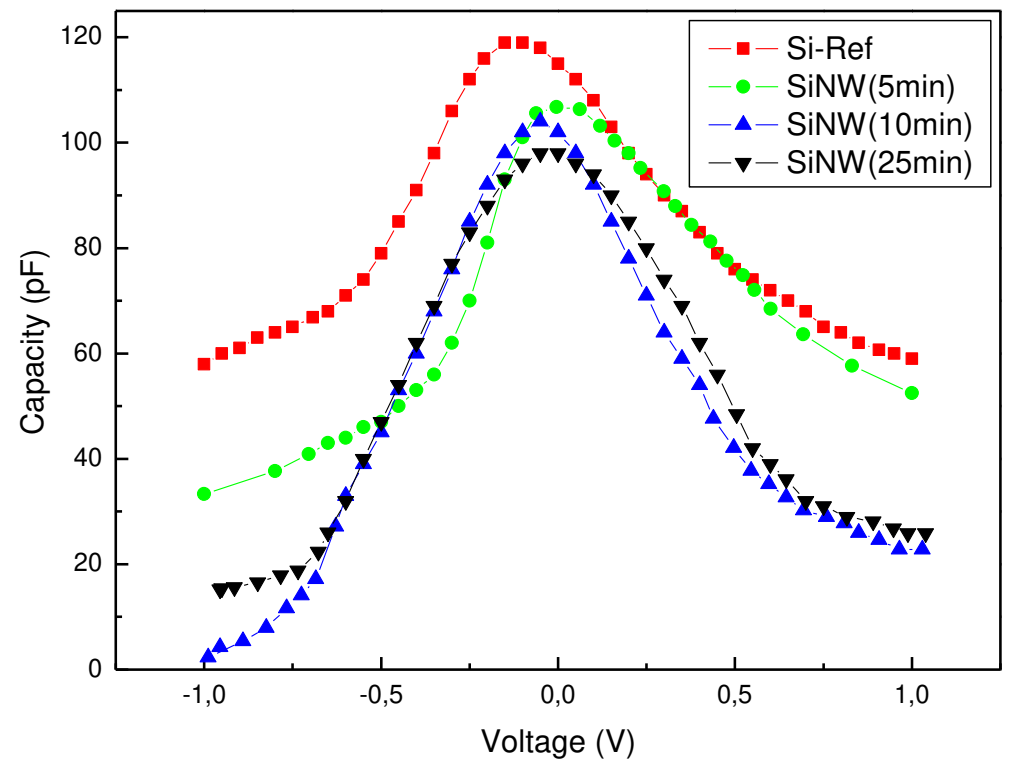

Fig.5 Capacitance-voltage plots of the Al/SiNWs heterojunction and Si-Ref at $1 \mathrm{MHz}$.

In order to calculate certain electrical parameters such as $\mathrm{N}_{\mathrm{A}}, \mathrm{V}_{\mathrm{d}}$ and $\Phi_{\mathrm{b}}$, the reverse biasC $\mathrm{C}^{-2}(\mathrm{~V})$ plots were drawn using the reverse and forward bias $\mathrm{C}-\mathrm{V}$ data and they are shown in Fig. 6 for SiNWs and Si-Ref. As seen in Fig. 6, $\mathrm{C}^{-2}$ versus $\mathrm{V}$ shows a nonlinear behavior, this can be explained by several reasons such as the oxidation of the samples, the lack of interfaces, the tunnel effect or the presence of deep acceptors [22]. Moreover, this nonlinear $1 / \mathrm{C}^{2}$ curves means that the carrier concentration will not be constant at the depletion layer.

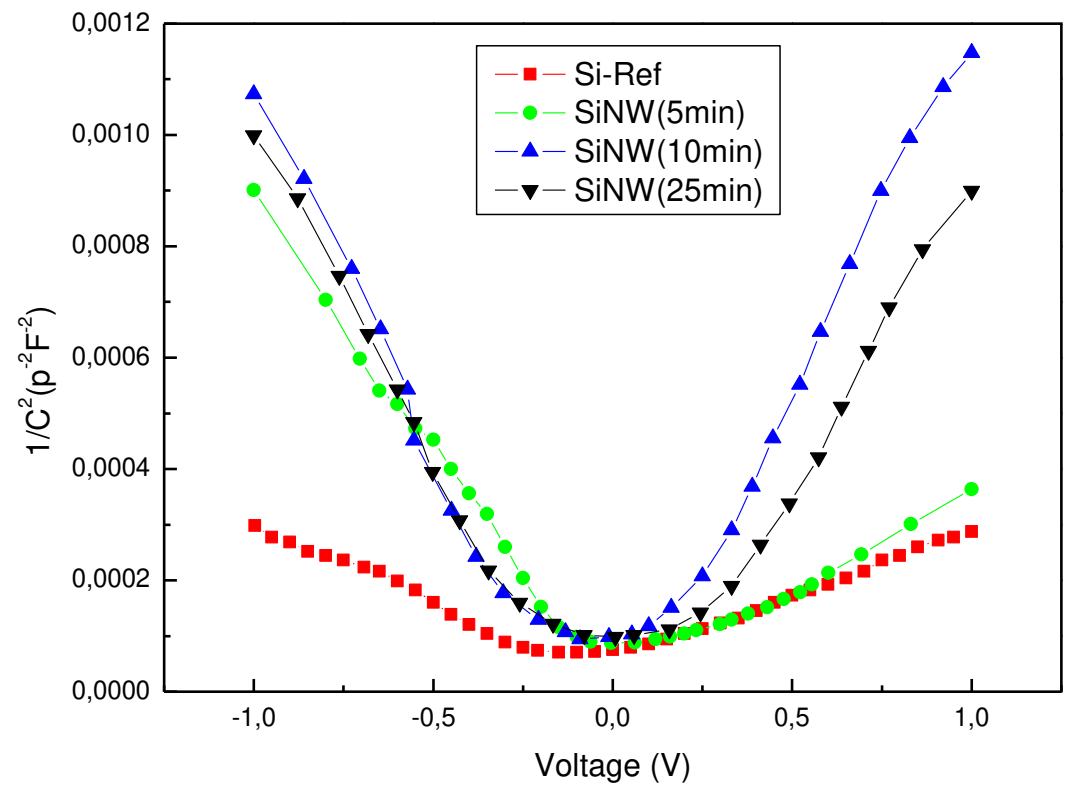

Fig.6 Capacitance-voltage plots of the Al/SiNWs and Al/Si diodes at $1 \mathrm{MHz}$. 
The barrier heights of our structures were determined from the capacitance-voltage measurements at $1 \mathrm{MHz}$ using the following equation.

$$
\Phi_{\mathrm{B}}=\mathrm{V}_{\mathrm{d}}+\mathrm{V}_{\mathrm{p}}
$$

Where $V_{p}$ is the potential difference between the Fermi energy level $\left(E_{f}\right)$ and the top of the valance band in the neutral region of $\mathrm{p}-\mathrm{Si}$, which is directly equal to $\mathrm{E}_{\mathrm{f}}$ and can be calculated once the carrier concentration $\mathrm{N}_{\mathrm{A}}$ is obtained. $\mathrm{V}_{\mathrm{p}}$ value was obtained from the following relation:

$$
\mathrm{qV}_{\mathrm{p}}=\mathrm{kT} \ln \left(\frac{N_{v}}{N_{A}}\right)
$$

Where, $\mathrm{N}_{\mathrm{V}}$ value is $1.04 \times 10^{19} \mathrm{~cm}^{-3}$ at $300 \mathrm{~K}$ [23]: the effective density of states in Si valance band.

The $V_{d}$ is the voltage intercept at $1 / C^{2}=0$ of the inverse of $1 / C^{2}$ versus $V$ plot. The obtained values of $V_{d}$ are given by the nearly straight part of the curve between $(0.3 \mathrm{~V}$ and $0 \mathrm{~V}$ for forward bias and between $-0.3 \mathrm{~V}$ and $0 \mathrm{~V}$ for reverse bias. It was found to be $0.25 \mathrm{~V}$ for the $\mathrm{p}$-Si and varied from 0.2 to 0.3 for the SiNWs etched at different times. Moreover the barrier heights, obtained from inverse of $\mathrm{C}^{2}-\mathrm{V}$ plot using equations 7,8, were found to be between 0.62 and $0.76 \mathrm{~V}$, these values are slightly higher than the values determined from the (I-V) characteristics for both the contacts. The lower value of $\Phi_{\mathrm{B}}$ deduced from forward (I-V) characteristics might be due to the image force lowering of the barrier height whereas the value obtained from the capacitance measurement is not affected by image force explained by Chang and Sze [24]. The presence of surface states may also be responsible for the low value of $\Phi_{\mathrm{B}}$ obtained from $\mathrm{I}-\mathrm{V}$ curve. All the results determined by the C-V method for forward and reverse polarization are summarized in Table 2.

Table 2 Electrical parameters obtained from C-V measurements of Al/SiNWs/p-Si//Al structures at room temperature in dark.

\begin{tabular}{ccccc}
\hline Samples & \multicolumn{2}{c}{ Forward bias } & \multicolumn{2}{c}{ Reverse bias } \\
\cline { 2 - 5 } & $\mathrm{V}_{\mathrm{d}}(\mathrm{V})$ & $\Phi_{\mathrm{b}}(\mathrm{eV})$ & $\mathrm{V}_{\mathrm{d}}(\mathrm{V})$ & $\Phi_{\mathrm{b}}(\mathrm{eV})$ \\
Si-Ref & 0.4 & 0.65 & 0.25 & 0.62 \\
SiNW (5min) & 0.32 & 0.69 & 0.15 & 0.68 \\
SiNW (10min) & 0.3 & 0.73 & 0.32 & 0.71 \\
SiNW (25min) & 0.2 & 0.76 & 0.3 & 0.75 \\
\hline
\end{tabular}

Slope of the linear fit of plot of $1 / \mathrm{C}^{2}$ as a function of reverse bias $\mathrm{V}$ relates to the effective carrier concentration, $\mathrm{N}_{\mathrm{A}}$, and is given by:

$$
\begin{gathered}
\text { Slope }=\frac{d\left(C^{-2}\right)}{d V}=-\frac{2}{e \varepsilon_{r} \varepsilon_{0} N_{A} A^{2}} \\
C=\frac{\varepsilon_{r} \varepsilon_{0} A}{W}
\end{gathered}
$$

The doping is not homogeneous, the $\mathrm{C}^{2}-\mathrm{V}$ curve is no longer a straight line and the differential capacitance method can be used to extract $\mathrm{N}_{\mathrm{A}}$. From relations (9) and (10), we can determine the doping profile in the desertion zone by plotting the variation of $\mathrm{N}_{\mathrm{A}}$ as a function of $\mathrm{W}$ (Fig.7). It has to be mentioned that the standard 
relations are derived under the assumption that a small ac voltage modulates only charges at the depletion layer edge [25].This dopant profile extraction method is based on the depletion approximation [26].The nanowire is depleted radially starting at the semiconductor surface as the bias scans from negative values (p-type). From a general point of view, all the samples in Fig.7 exhibit a high concentration $2.5 \times 10^{16} \mathrm{~cm}^{-3}$, that sharply decreases to $10^{15} \mathrm{~cm}^{-3}$ within the initial of the Si substrate. The measured depth profile shows confinement of carriers at the hetero interface and the carrier concentration rapidly falls off with increasing depth [27]. This decrease was slower in the nanowire structures with a variation of doping that extends over a width of a few hundred nm. The upward shift of the calculated concentrations at the surface (first point in Fig.7for all samples) is probably due to the presence of a native oxide at the sample surface that may result in a different electrostatic configuration of the surface, hence the effective quantification of concentration in the depth profile is not reliable in the so-called transient region, i.e.in the region close to the surface of the sample. Whereas, the increase of the doping concentration on the right side of Fig.7 is likely due to diffusion from the substrate [28].

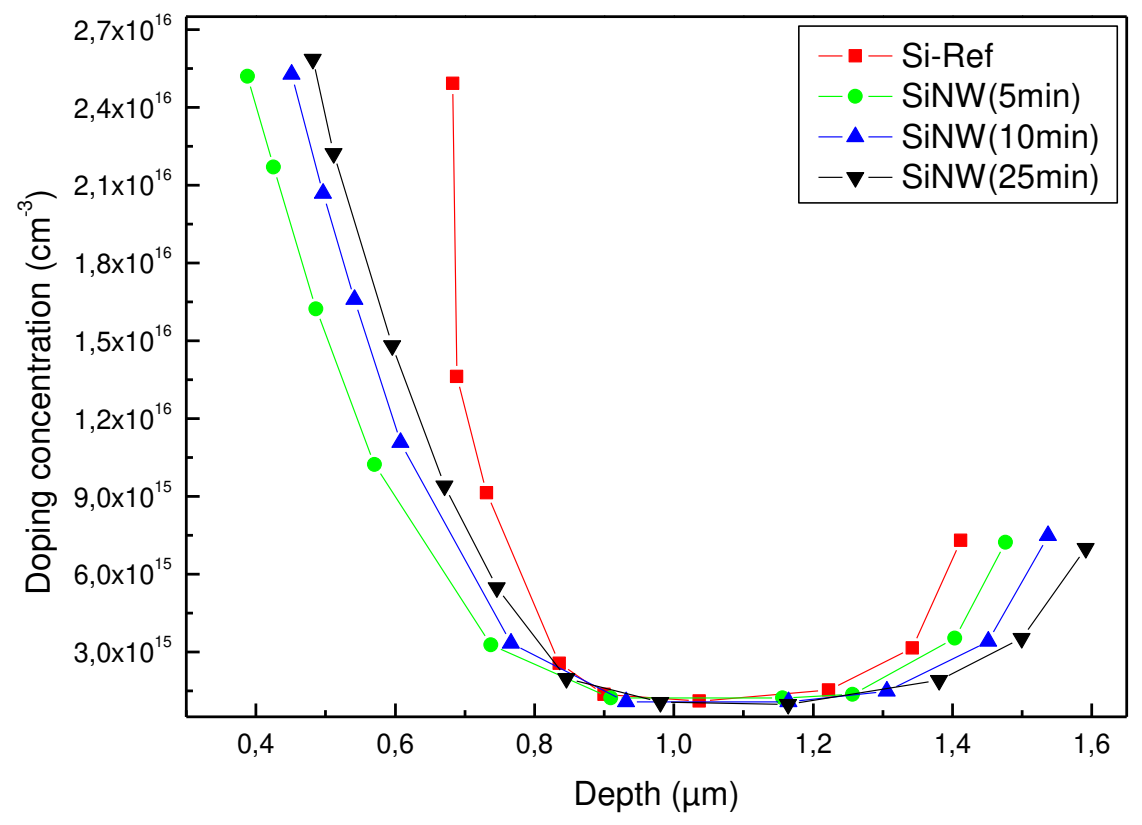

Fig.7 Doping profile as a function of depth in samples etched at different times and the reference substrate.

\section{Conclusion}

In summary, we reported the fabrication of high quality of SiNWs through the metal (Ag) assisted chemical etching method (MACE) at various etching time. This method is introduced to control the morphology of silicon nanowire arrays. A successfully Al/SiNWs hetero junction diodehas been formed. Symmetric (I-V) curves were obtained due to the different barrier heights at the two sides of the SiNWs. A metal-semiconductor-metal model and a thermionic field emission theory were used to analyze the (I-V) and (C-V) characteristics. According to the electric parameters values determined by the (I-V) characteristics, the electron transport behavior is explained by the thermionic field emission method (TFE) which added the effect of the tunneling current compared to the conventional thermionic emission theory. The voltage dependence of the capacity confirms the existence of 
interface states, the re-structuring and the re-ordering of surface charge under the etching effect.The tunnel effect or the presence of deep acceptors leads to the variation of the carrier concentration at the depletion layer.In addition, the doping profile determined after $(\mathrm{C}-\mathrm{V})$ curves is variable over a few hundred of nm, which is probably due to the etching operations at the surface. Finally, these electrical characterization shows the performance of SiNWs prepared with the MACE method possess coherent electrical transport properties with their corresponding starting wafer Si-Ref and may help advanced realization of Si nanostructure-based high performance devices. This study can be the guidance to correct measurement and understanding of the contact transport, which is useful for NWs device design and fabrication. The detailed studies of transport through silicon nanowires are expected to open up new opportunities towards potential applications of semiconductor nanowires in nanoelectronic, nano photonic, and Nano spintronic devices.

\section{Acknowledgments}

We wish to acknowledge the support of the authors in offering suggestions and encouragement, testing new versions.

\section{References}

1. O.Gunawan, S.Guha, Characteristics of vapor-liquid--solid grown silicon nanowire solar cells. Sol. Energy Mater Sol. Cells93(8), 1388-1393 (2009). https://doi.org/10.1016/j.solmat.2009.02.024

2. L.Hu, G. Chen, Analysis of optical absorption in silicon nanowire arrays for photovoltaic applications. Nano Lett7 (11), 3249-3252 (2007). https://doi.org/10.1021/n1071018b

3. M. M.Adachi, M. P.Anantram, K. S. Karim, Core-shell silicon nanowire solar cells. Sci. Rep.3(1), 1-6(2013). https://doi.org/ 10.1038/srep01546

4. T.Onuma, S.Fujioka, T.Yamaguchi, Y.Itoh, M.Higashiwaki, K.Sasaki, T.Honda, Polarized Raman spectra in B-Ga2O3 single crystals. J. Cryst. Growth401, 330-333(2014). https://doi.org/10.1016/j.jcrysgro.2013.12.061

5. W. M. Cho, Y. J. Lin, H. C.Chang, Y. H. Chen, Electronic transport for polymer/Si-nanowire arrays/n-type Si diodes with and without Si-nanowire surface passivation. Microelectron. Eng.108, 24-27(2013). https://doi.org/10.1016/j.mee.2013.03.074

6.C. Thelander, P. Agarwal, S. Brongersma, J. Eymery, L. F. Feiner, A. Forchel, L. Samuelson, Nanowire-based one-dimensional electronics. Mater. Today9(10), 28-35(2006). https://doi.org/10.1016/S1369-7021(06)71651-0

7. W.Wang, D. Li, M. Tian, Y. C. Lee, R. Yang, Wafer-scale fabrication of silicon nanowire arrays with controllable dimensions. Appl. $\quad$ Surf. $\quad$ Sci.258(22), 8649-8655(2012). https://doi.org/10.1016/j.apsusc.2012.05.067

8. C. Zhang, S. Li, W. Ma, Z. Ding, X. Wan, J.Yang, J. Qiu, Fabrication of ultra-low antireflection SiNWs arrays from mc-Si using one-step MACE. J Mater Sci - Mater El28(12), 8510-8518(2017). https://doi.org/ 10.1007/s10854-017-6573-7 
9. Y. Qi, Z. Wang, M. Zhang, X. Wang, A. Ji, F. Yang, Electron transport characteristics of silicon nanowires by metal-assisted chemical etching. AIP Adv.4(3), 031307(2014). https://doi.org/10.1063/1.4866578

10. A. Svizhenko, P. W. Leu, K. Cho, Effect of growth orientation and surface roughness on electron transport in silicon nanowires. Phys. Rev. B75(12), 125417(2007). https://doi.org/10.1103/PhysRevB.75.125417

11.C. Amri, R. Ouertani, A. Hamdi, H.Ezzaouia, Effect of acid vapor etching on morphological and opto-electric properties of flat silicon and silicon nanowire arrays: A comparative study. Superlattices Microstruct.91, 278289(2016). https://doi.org/10.1016/j.spmi.2016.01.007

12. C. Amri, R. Ouertani, A. Hamdi, H.Ezzaouia, Effect of Silver-Assisted Chemical Vapor Etching on morphological properties and silicon solar cell performance. Mater Sci Semicond Process63, 176-183(2017). https://doi.org/10.1016/j.mssp.2017.02.019

13. D. V. GEPPERT, A. M. COWLEY, B. V. DORE, Correlation of Metal- Semiconductor Barrier Height and Metal Work Function; Effects of Surface States. J. Appl. Phys.37, 2458-2467(1966). https://doi.org/10.1063/1.1708837

14. H. C. CARD, E. H. RHODERICK, Studies of tunnel MOS diodes I. Interface effects in silicon Schottky diodes. J. Phys. D: Appl. Phys.4(10), 1589 (1971). http://iopscience.iop.org/0022-3727/4/10/319

15. K. Sarpatwari, N. S. Dellas, O. O. Awadelkarim, S. E. Mohney, Extracting the Schottky barrier height from axial contacts to semiconductor nanowires. Solid State Electron54(7), 689-695(2010). https://doi.org/10.1016/j.sse.2010.03.006

16. İ. H.Taşdemir, Ö.Vural, İ.Dökme, Electrical characteristics of p-Si/TiO2/Al and p-Si/TiO2-Zr/Al Schottky devices. Philos Mag (Abingdon)96(16), 1684-1693(2016). https://doi.org/10.1080/14786435.2016.1178403

17. M. Zhu, J. Zhang, Z. Wang, L. Wan, X.Chen, Effect of interfacial layer and series resistance on electrical characteristics for the PtSi/p-SiNWs Schottky diode. Physica E Low Dimens. Syst. Nanostruct. 43(1), 515520(2010). https://doi.org/10.1016/j.physe.2010.09.009

18. Z. Y.Zhang, C. H. Jin, X. L. Liang, Q. Chen, L. M. Peng, Current-voltage characteristics and parameter retrieval of semiconducting nanowires. Appl. Phys. Lett.88(7), 073102(2006). https://doi.org/10.1063/1.2177362

19. C. H. Kuo, J. M. Wu, S. J. Lin, Room temperature-synthesized vertically aligned InSb nanowires: electrical transport and field emission characteristics. Nanoscale Res. Lett.8(1), 69(2013). https://doi.org/10.1186/1556276X-8-69

20. B. N. Naik, L. Agarwal, S.Tripathi, Microstructural and Electrical Characterization of Pt/Si Nanowires Schottky Diode Grown by Metal Assisted Chemical Etching Method. Int. J. Thin. Fil. Sci. Tec6(3), 107111(2017). http://dx.doi.org/10.18576/ijtfst/060302

21. Y. Qi, Z. Wang, M. Zhang, X. Wang, A. Ji, F.Yang, Electron transport characteristics of silicon nanowires by metal-assisted chemical etching. AIP Adv.4(3), 031307(2014). https://doi.org/10.1063/1.4866578 
22. B. Sermage, Z. Essa, N. Taleb, M. Quillec, J. Aubin, J. M. Hartmann, M. Veillerot, Electrochemical capacitance voltage measurements in highly doped silicon and silicon-germanium alloys. J. Appl. Phys.119(15), 155703(2016). https://doi.org/10.1063/1.4946890

23. D.Lichtenberger, K. Ellmer, R. Schieck, S. Fiechter, H. Tributsch, Structural, optical and electrical properties of polycrystalline iron pyrite layers deposited by reactive dc magnetron sputtering. Thin Solid Films 246(1-2), 612(1994). https://doi.org/10.1016/0040-6090(94)90723-4

24.C. Y.Chang, S. M. Sze, Carrier transport across metal-semiconductor barriers. Solid State Electron 13(6), 727-740(1970). https://doi.org/10.1016/0038-1101(70)90060-2

25. Y.Elogail, E. Kasper, F.Gunzer, A. Shaker, J. Schulze, Investigation of capacitance voltage characteristics of strained $\quad \mathrm{Si} / \mathrm{SiGe} \quad \mathrm{n}$-channel MODFET varactor. Solid State Sci.56, 73-78(2016). https://doi.org/10.1016/j.solidstatesciences.2016.04.007

26. E. C. Garnett, Y. C. Tseng,D. R.Khanal, J. Wu,J.Bokor, P. Yang, Dopant profiling and surface analysis of silicon nanowires using capacitance-voltage measurements. Nat. Nanotechnol.4(5), 311(2009). https://doi.org/ 10.1038/NNANO.2009.43

27.P.Ranga,A.Rishinaramangalam, J. Varley, A. Bhattacharyya,D.Feezell, S. Krishnamoorthy, Si-doped $\beta$-(A10. 26Ga0. 74) $2 \mathrm{O} 3$ thin films and heterostructures grown by metalorganic vapor-phase epitaxy. Appl. Phys. Express12(11), 111004(2019). https://doi.org/10.7567/1882-0786/ab47b8

28. E.Thor, M.Mühlberger, L.Palmetshofer, F.Schäffler, Deep-level transient spectroscopy of dislocation-related defects in epitaxial multilayer structures. J. Appl. Phys.90(5), 2252-2256(2001). https://doi.org/10.1063/1.1389762 
Figures

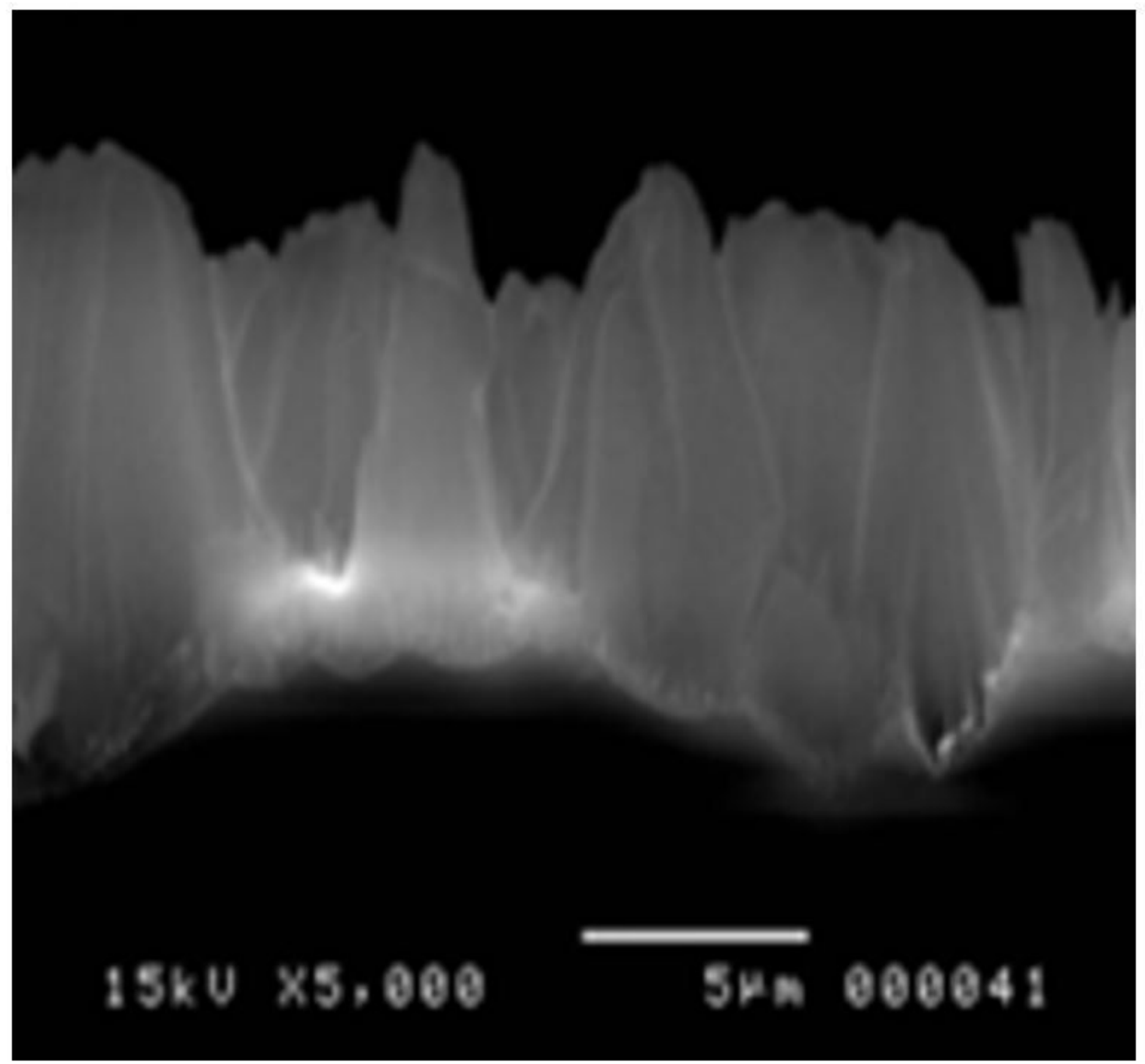

Figure 1

Cross section SEM images of the silicon nanowires. 


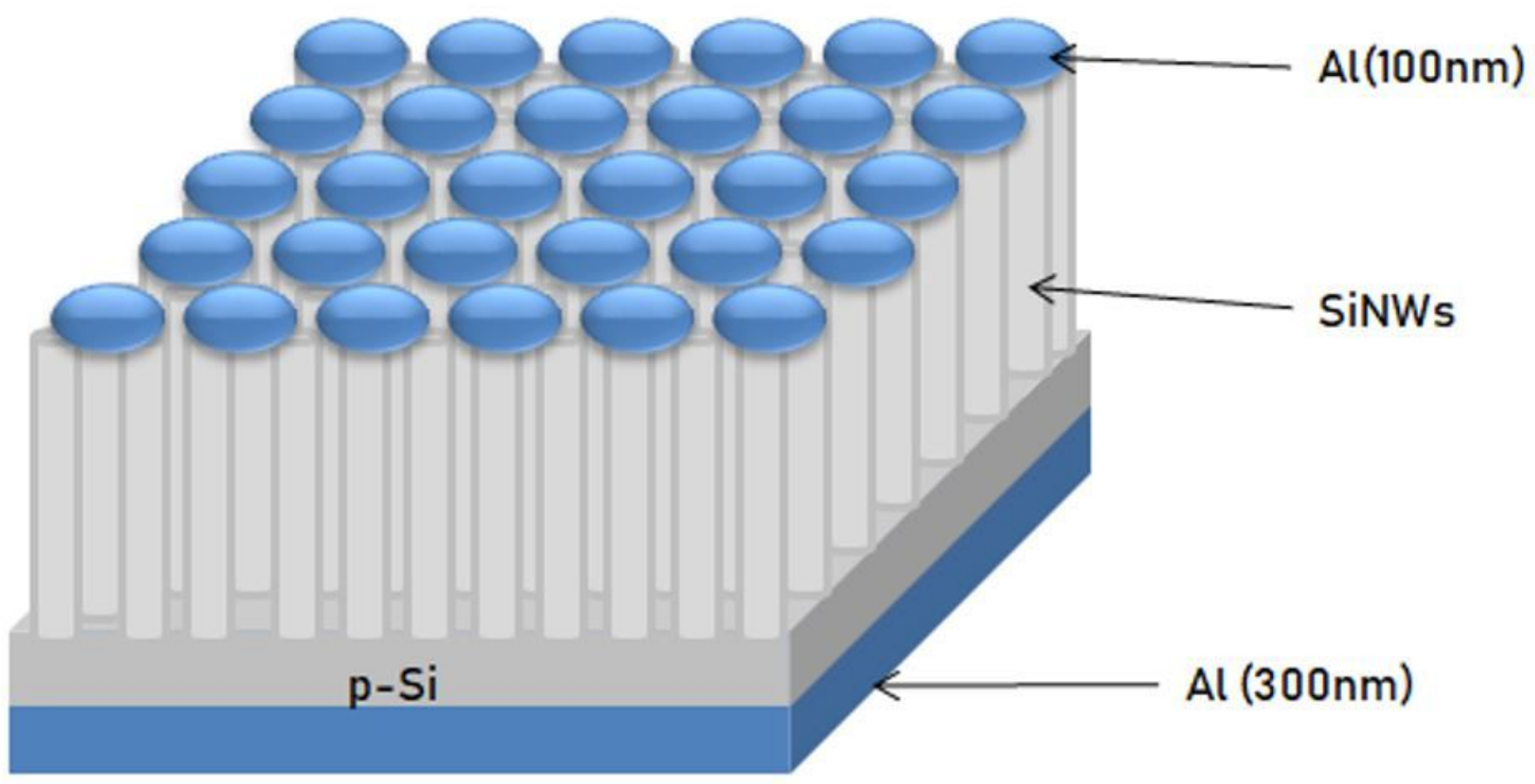

Figure 2

Schematic illustration of the device used in the $\mathrm{I}-\mathrm{V}$ and $\mathrm{C}-\mathrm{V}$ measurements. 


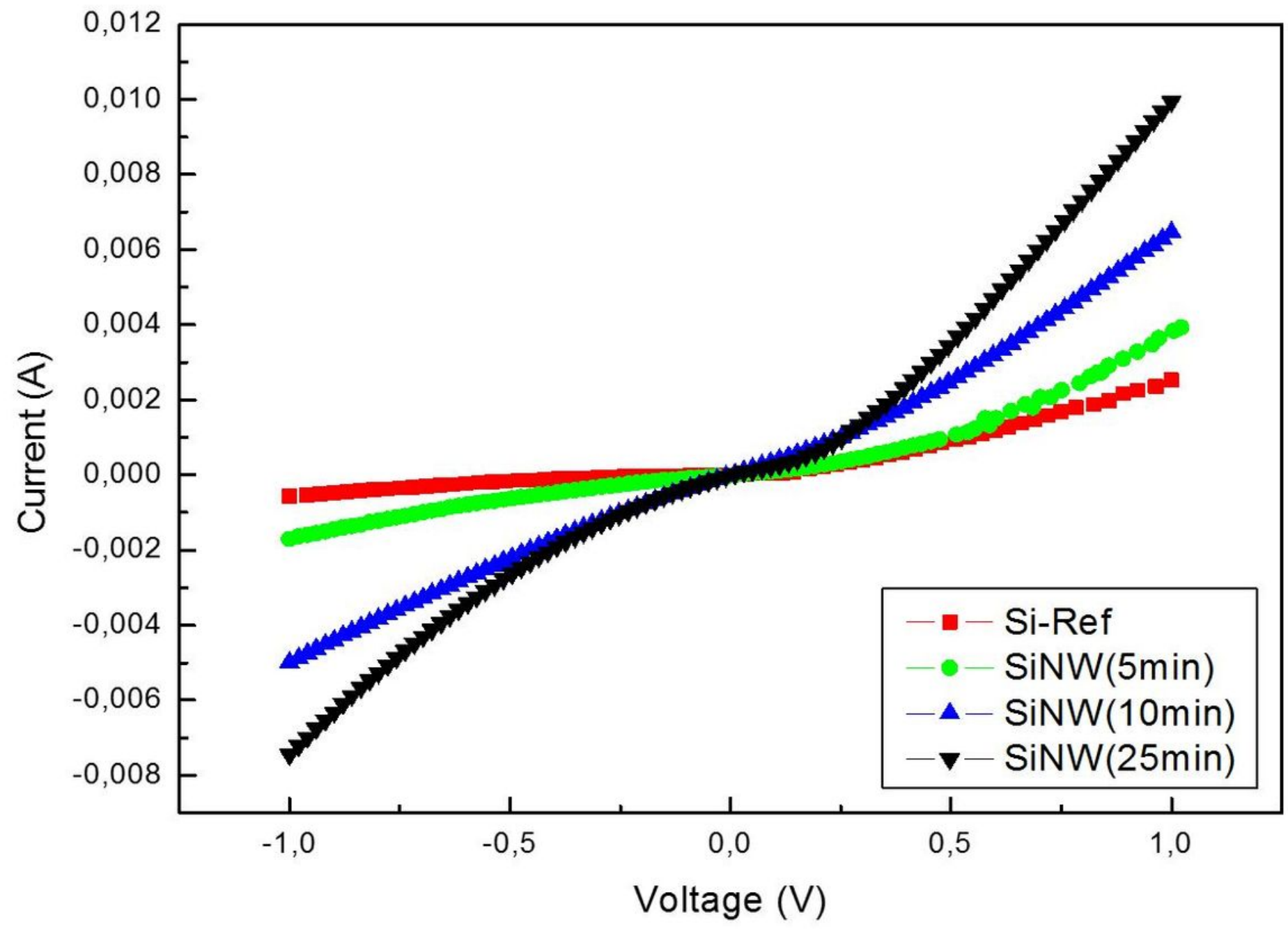

Figure 3

Experimental I-V characteristics of the Si substrate as reference sample and SiNWs structures obtained in dark and at room temperature. 


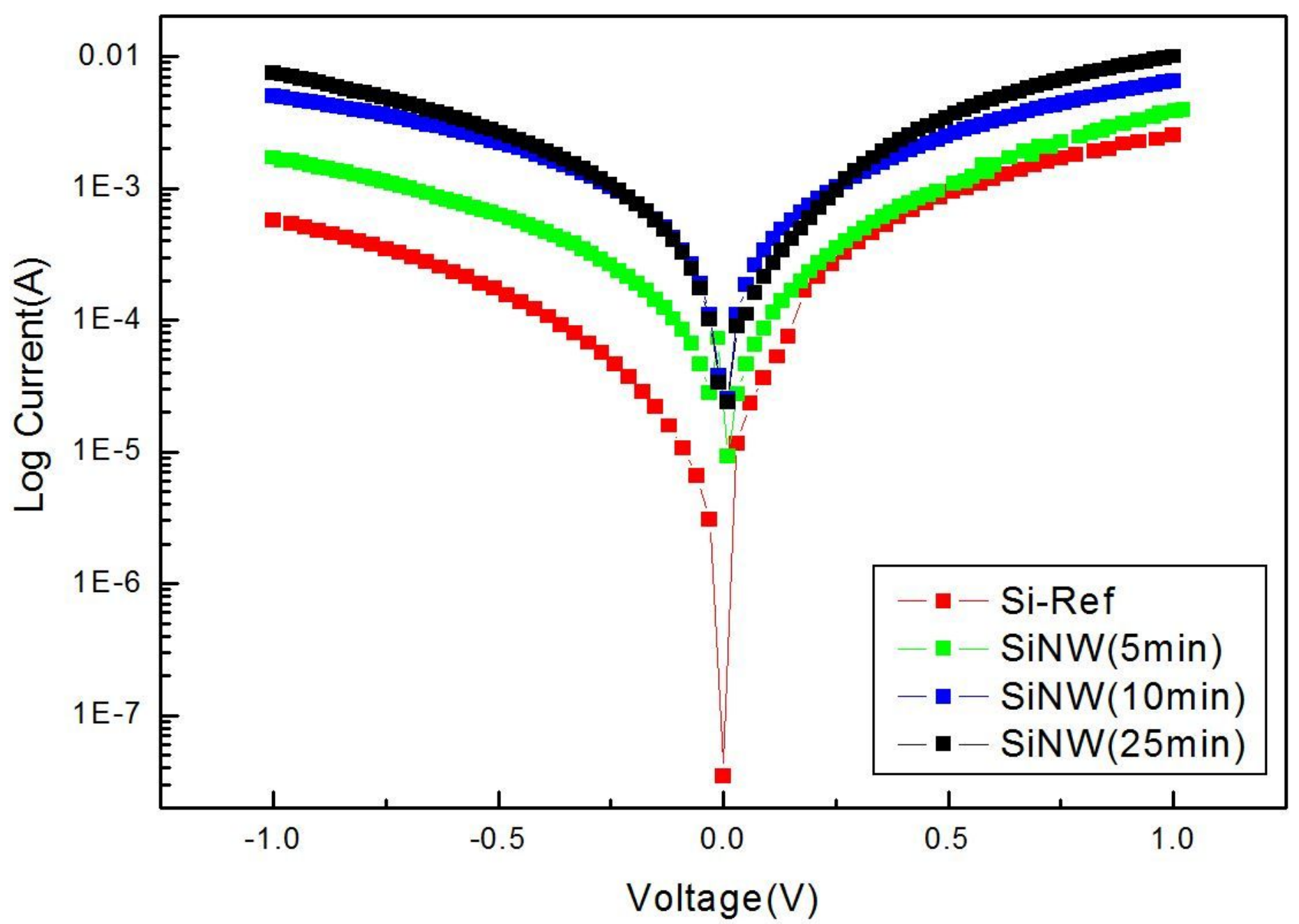

Figure 4

Experimental semi-logarithmic logl-V characteristics of the c-Si as reference sample and SiNWs structures at room temperature. 


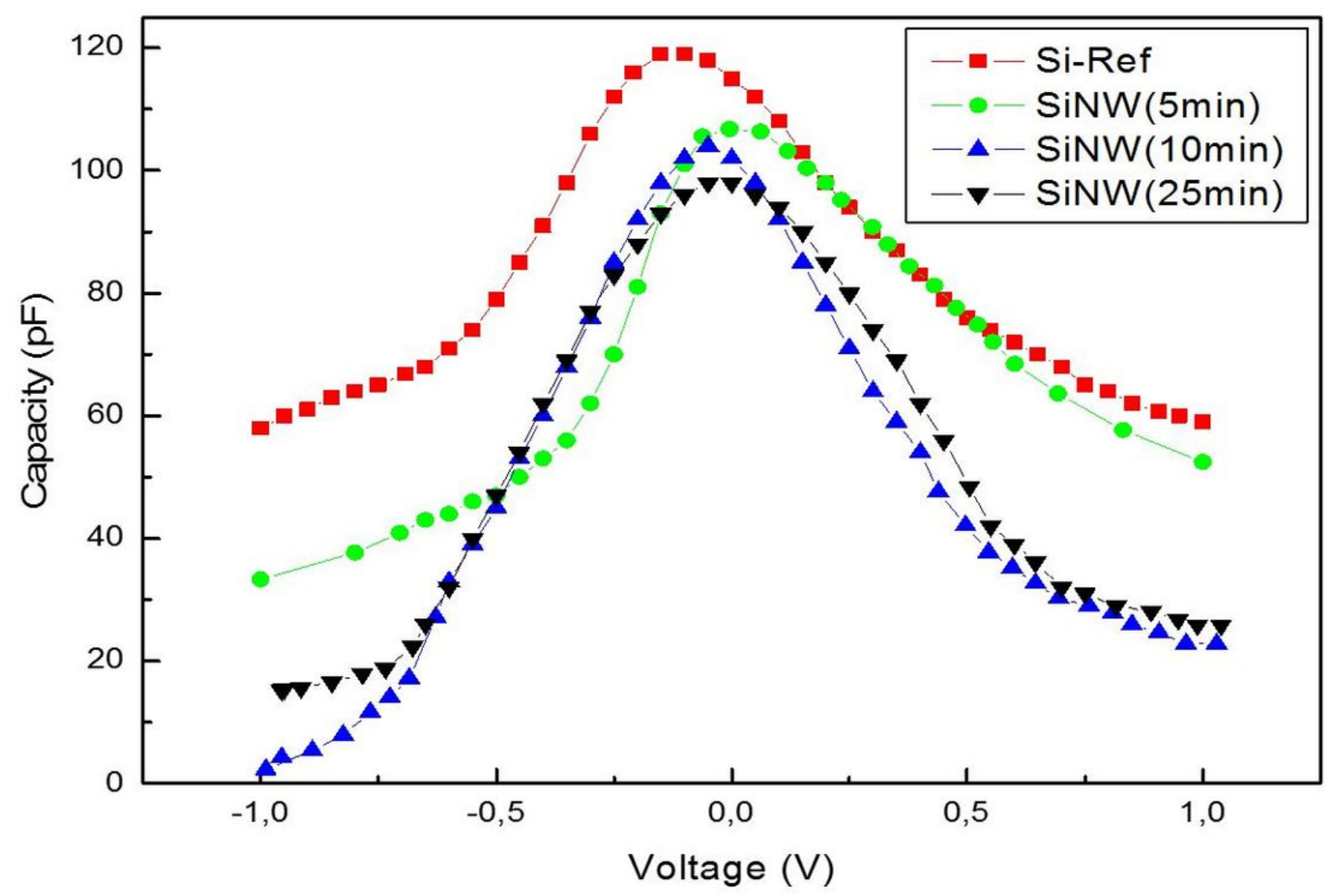

Figure 5

Capacitance-voltage plots of the Al/SiNWs heterojunction and Si-Ref at $1 \mathrm{MHz}$. 


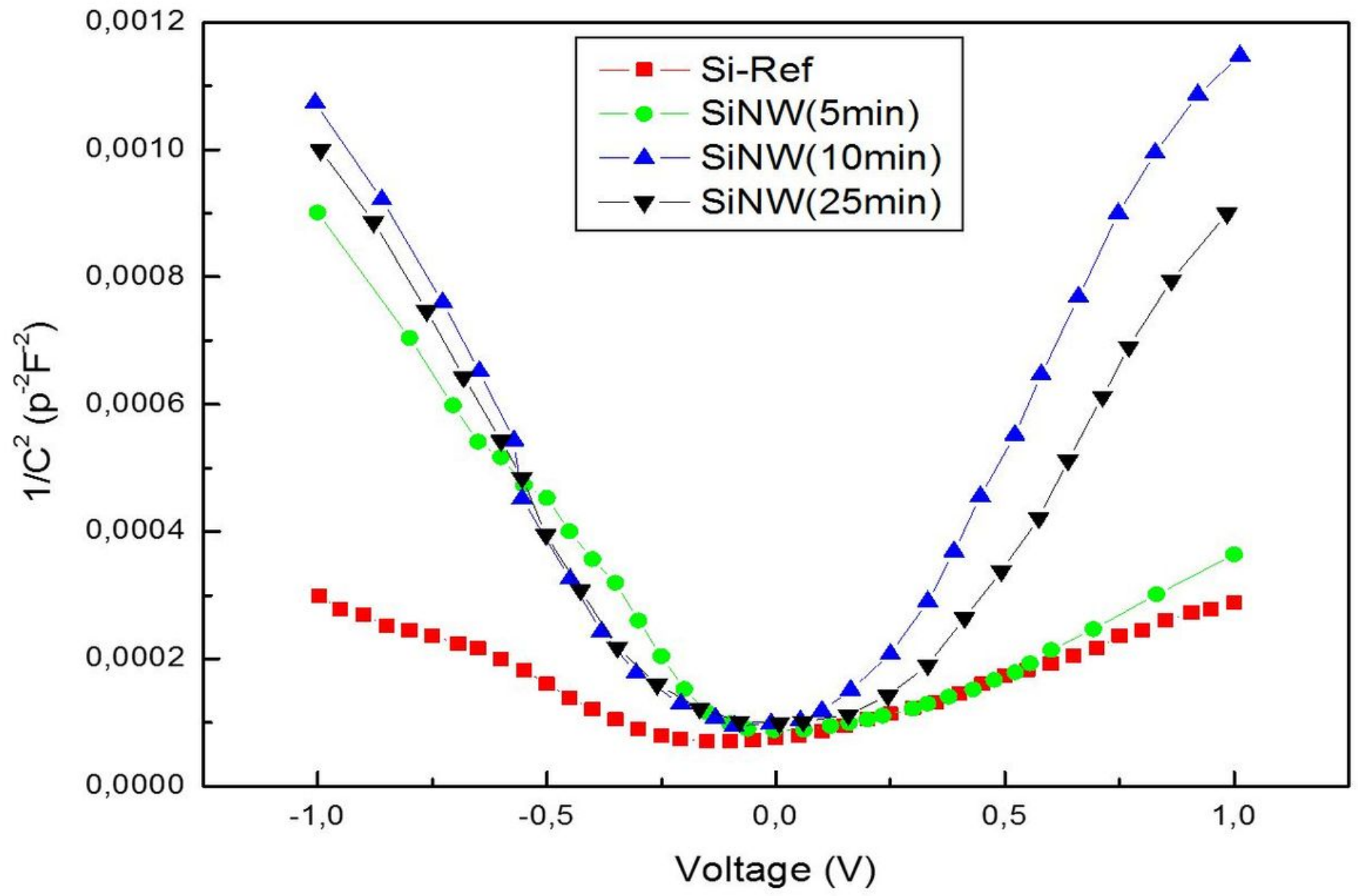

Figure 6

Capacitance-voltage plots of the $\mathrm{Al} / \mathrm{SiNWs}$ and $\mathrm{Al} / \mathrm{Si}$ diodesat $1 \mathrm{MHz}$ 


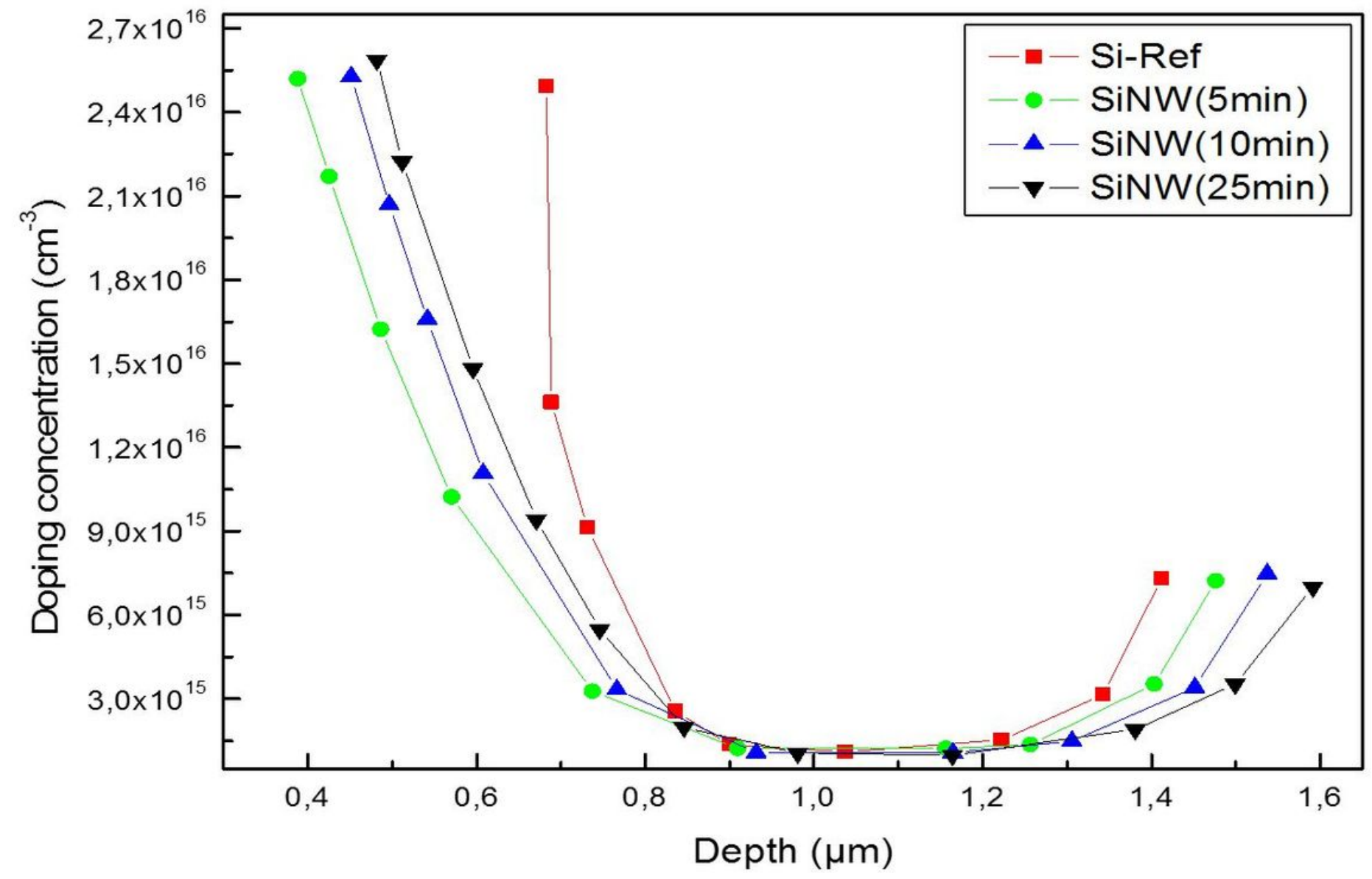

Figure 7

Doping profile as a function of depth in samples etched at different times and the reference substrate. 